Pecvnia, 9 (2009), pp. 225-245

\title{
El carácter empírico y lógico de la contabilidad
}

Recibido: Diciembre 2009

Aceptado: Enero 2010

\author{
Eduardo R. Scarano \\ scarano@econ.uba.ar \\ Universidad de Buenos Aires \\ Fac. de Ciencias Económicas-CIECE \\ Av. Córdoba 2122 \\ (1120) Buenos Aires (Argentina)
}

El objetivo de este artículo es argumentar acerca del carácter esencialmente empírico y científico de la contabilidad. Este punto de vista implica rechazar el carácter predominantemente normativo que posee la contabilidad, por lo menos, en Latinoamérica. Se considera que el normativismo de cualquier tipo es meramente secundario.

La contabilidad es una disciplina científica, pero al carecer de leyes estrictas no constituye una ciencia básica, es una tecnología social.

Se mostrará que tiene una estructura axiomática específica que se puede exponer con la lógica estándar, lo mismo que la matemática contable que supone la partida doble.

Palabras clave: contabilidad empírica, metodología contable, regulacionismo, estatus de la contabilidad, axiomática contable.
The aim of this article is to argue about the essentially empirical and scientific character of the accounting. This point of view implies rejecting the predominantly normative character that possesses the accounting, at least, in Latin America. It thinks that the normativism of any type is merely secondary.

The accounting is a scientific discipline, but it lacks strict laws, therefore, it does not constitute a basic science, is a social technology. It will appear that it has an axiomatic specific structure that can be exposed by the standard logic the same as the accounting mathematics that supposes the double entry.

Key words: empirical accounting, accounting methodology, regulationism, status of the accounting, axiomatic accounting. 
En este artículo se abordan algunas cuestiones de tipo metodológico acerca de la contabilidad. Esta indagación es sumamente interesante pues en un primer análisis esta disciplina presenta características singulares que la diferencian de las restantes. Así, la expresión de las soluciones contables mediante normas no es común en otras disciplinas o el tratamiento de la información mediante partida doble; son aspectos que merecen analizarse desde un punto de vista metodológico.

Se argumenta en favor de la naturaleza empírica de la contabilidad y, por lo tanto, del carácter subordinado del enfoque normativo. Para complementar el análisis anterior, pero también para plantear la naturaleza y características de la partida doble y la matemática subyacente, se presenta y analiza una formalización axiomática. La elucidación de los conceptos y los problemas relacionados con estos análisis conducen naturalmente a indagar el estatuto epistemológico de la contabilidad. Se defiende su naturaleza principalmente tecnológica y, por tanto, científica, lo cual no excluye características secundarias que pudieran atribuírsele -técnica, arte.

Cualquiera de estos problemas tiene innumerables precedentes y soluciones, tanto históricas como contemporáneas. Aquí la novedad no reside en los problemas y en las evaluaciones de las soluciones, sino en el tipo de argumentos con los cuales se defienden.

En la sección I se aborda el carácter empírico de la contabilidad y se analiza el papel del normativismo; en II se presenta una formalización axiomática que permite analizar la naturaleza de los enunciados -empíricos o normativos- y de la partida doble; en III se desarrolla el estatuto tecnológico de la contabilidad y la singularidad de su desarrollo histórico reciente entre las disciplinas; finalmente, en IV se exponen las conclusiones.

\section{EL CARÁCTER EMPÍRICO}

\section{Las modalidades normativas de la contabilidad}

La contabilidad, especialmente en Latinoamérica, se presenta como un conjunto de normas, sin embargo, este enfoque normativo ni ha predominado a lo largo de la historia de la contabilidad, ni constituye el núcleo de las cuestiones contables. 
A título meramente ilustrativo en Argentina la actividad de los contadores está reglada mediante normas de diferente jerarquía. Sin pretender ser exhaustivos podemos enumerar: el Código de Comercio, Leyes (como la N¹9550/72 entre otras y su respectivo Decreto Reglamentario), disposiciones de Organismos Públicos como la Comisión Nacional de Valores, el Banco Central de la República Argentina, la Inspección General de Justicia, la Bolsa de Comercio de Buenos Aires, la de diferentes entes Públicos Provinciales. Las Leyes facultan a emitir normas específicas, las Resoluciones Técnicas, a los Consejos y Colegios Profesionales en Ciencias Económicas y a las respectivas Federaciones. Las normas emitidas por estos organismos profesionales así como las emitidas por los organismos mencionados precedentemente, se refieren a las condiciones $y$ procedimientos que debe cumplir y reunir el contador público cuando emite opinión sobre ciertos aspectos contables para terceros o produce información para uso externo. Esta normativa en su conjunto se refiere principalmente a los llamados N.A.G.A. (Normas de Auditoría Generalmente Aceptada), y a los P.C.G.A. (Principios de Contabilidad Generalmente Aceptados).

El núcleo de la actividad contable entendida normativamente consiste en la génesis, desarrollo y justificación de este conjunto normativo. La contabilidad puede considerarse normativa cuando sus enunciados más importantes son normas, es decir, la principal función de los enunciados contables no es informativa sino normativa. Existen tres maneras muy extendidas, aunque no únicas, de normativismo contable: el prescriptivismo, el consensualismo y el apriorismo.

Para el prescriptivismo las normas contables son de carácter promulgable o jurídico. Son normas que autorizan, obligan o prohíben de manera semejante a las jurídicas. A los prescriptivistas los distingue la concepción positiva de las normas: están justificadas si rigen. Toda otra cuestión es secundaria y habrá de derivarse al organismo que tiene la potestad de promulgarlas.

El consensualismo propone un proceso previo a la promulgación de la norma y, además, un procedimiento para cambiar la norma. En ambos aspectos resulta esencial el consenso de los profesionales, especialmente el de los contables más destacados. Este enfoque es el que denominó a las normas contables más importantes Principios de ... generalmente aceptados. Los consensualistas pensaban que la solución de los problemas contables se volvería más segura en la medida que reflejaran 
pautas y criterios de aceptación general (tomando en cuenta especialmente los referentes de la profesión).

El apriorismo, también denominado investigación contable a priori, consiste en sostener una norma contable mediante argumentaciones. Una clase de argumentaciones especial, del tipo que empleamos en la vida cotidiana cuando discutimos a cerca de la interpretación de un hecho, de perseguir tal o cual objetivo o de una posición política. Su característica más notable es no aplicar el método científico para la aceptación de las premisas o la conclusión.

identifican:

El normativismo posee varios rasgos comunes que lo

1. La clase de enunciados más importantes de la contabilidad son normas.

2. El dominio de los problemas es el de las cuestiones resolubles mediante normas; el resto de los problemas es periférico, por ej., la contabilidad gerencial.

3. La justificación de los enunciados se reduce a mostrar la vigencia de la norma o a sostenerlas mediante argumentaciones que no emplean el método científico.

4. Los criterios para cambiar los enunciados contables (las normas): aunque aceptan que cambian no tienen una teoría definida al respecto, oscilan entre el positivismo de la norma hasta el consenso o la autoridad para emitirla.

5. La naturaleza de la disciplina: la mayor parte se inclina por darle un estatus de técnica o arte y fundamentalmente de carácter jurídico.

La resolución de problemas de una manera normativa se relaciona con el proceso regulador de la actividad de los especialistas que se identifica con la emisión de normas. Con el aditamento que actualmente cada vez es menos importante considerarlos generalmente aceptados, basta tener la potestad legal para establecer las normas. Esta es la razón por la que se ha producido un significativo desplazamiento en la terminología, y se habla simplemente de normas profesionales. Aquí la justificación fundamental de una norma contable es la capacidad legal para emitirla. Todo otro fundamento o razonabilidad es facultativo y marginal. 
2. La historia de la contabilidad y el punto de vista normativo

El proceso regulador de la contabilidad se inició decisivamente en Gran Bretaña debido a las crisis suscitadas por la fiebre de las inversiones en ferrocarriles, entre 1830-70. El Parlamento sancionó un Acta que daba instrucciones detalladas acerca de la forma y el contenido de las cuentas; exigía la partida doble para la publicación de las cuentas y exigía uniformidad contable entre las compañías. Un Acta de 1840 obligaba a que un auditor verificase las cuentas 40 días antes de publicarse (Pollins 1956: 332, 336 y ss.; Edey y Panitpakdi 1956). Lo curioso es que Estados Unidos adopte, en este terreno liberal a ultranza, el regulacionismo que en contadas ocasiones será legal, a diferencia en ambos aspectos de Gran Bretaña. Las primeras manifestaciones reguladoras significativas en Estados Unidos surgen con el American Institute of Accounting (A.I.A.) que fue creado en 1917 por la American Association of Public Accountants. La A.I.A. difunde los Special Bulletins que pueden considerarse las primeras normas escritas de importancia. También publicó en 1918 un programa de procedimientos de auditoría. Para considerar los inicios y los desarrollos más significativos de los aspectos reguladores en ese país ver Moonitz (1970) y Zeff (1972).

La actividad reguladora, poco significativa, sufrió un giro decisivo debido a la crisis de 1929. La contabilidad resultó muy desacreditada por sus dictámenes. Empresas que desde el punto de vista contable eran empresas en marcha, iban a la quiebra. Los procedimientos contables hasta facilitaban las estafas. Todo esto conduce a una profundización de la crisis de la contabilidad y a la revisión de la disciplina que finaliza en una concepción normativista.

En 1930 el A.I.A. y la Bolsa de New York crean un organismo permanente para considerar las normas y procedimientos contables. En 1933 publican la correspondencia intercambiada y aparecen por primera vez los PCGA, Principios de Contabilidad Generalmente Aceptados. La autoridad de estos principios se fue generando con el tiempo. Entre 193034 se consolida la estructura del sistema mediante una regulación de carácter profesional, controlada desde el sector público, para las sociedades que cotizan en Bolsa, por la S.E.C. (Security Exchange Commission). Este organismo es de carácter público. Posee competencia específica para modificar o emitir regulaciones sobre estados financieros que comúnmente adopta tras analizar los principios emanados de los organismos profesionales. Esta regulación contable se fundamenta básicamente en dos aspectos, el primero, continuar con lo que se practica, los principios de contabilidad 
generalmente aceptados; el segundo, institucionalizar esta práctica a través de una declaración de un organismo o institución reguladora.

Como señalamos en el punto anterior, la regulación se puede reforzar con un soporte legal, por ejemplo en Argentina, que habilita por ley a un organismo -en ese país a los colegios profesionales- a emitir normas contables.

3. El núcleo de la contabilidad no consiste en normas sino en hechos. Las soluciones contables están encapsuladas en normas

La contabilidad normativa no aplica el modelo científico en la resolución de sus problemas. Para enfatizar que no utiliza el criterio de contrastación con la realidad como criterio decisivo en la evaluación de una solución a un problema, ha sido calificada de a priori. La solución se sostiene independientemente del cotejo sistemático y controlado con la experiencia.

El método normativo consta básicamente de dos pasos según señalamos anteriormente. El primero consiste en recoger la práctica existente para resolver un problema. Puede hacerse sometiendo una norma existente a consulta abierta de los contadores, o efectuando consultas a los contadores considerados relevantes, o por otros medios. La práctica recogida se discute e incluso se modifica. Normalmente el encargado de llevar adelante esta tarea y proponer una norma es un organismo especializado de la profesión compuesto por especialistas.

El segundo es institucionalizar una práctica para resolver un problema. Un organismo representativo de la profesión o con autoridad para hacerlo emite normas profesionales, normalmente denominadas en Argentina Resoluciones Técnicas. Como se ha señalado antes, esta declaración puede llegar a tener fuerza legal. Incluso cuando es legal no obliga incondicionalmente, es posible actuar de otra manera. Este comportamiento es aceptable siempre y cuando quien no se ajuste a la norma explique las razones de su proceder y el organismo profesional las considere plausibles.

Este método de resolución de problemas ha tenido éxitos. Por sólo citar uno, cuando Argentina en los 70 sufrió un proceso inflacionario tan persistente y notorio que el criterio de valuación por costo histórico no se podía sostener, la profesión contable respondió con una solución para valuar bienes en contextos inflacionarios. Al modelo se lo puede 
criticar pero no sobre la base de afirmar que no encuentra soluciones, o quizás buenas soluciones. Tampoco se lo puede desestimar porque conduzca sistemáticamente a adoptar soluciones dogmáticas. Señalamos que las prácticas aparentemente exitosas se recogen, se comparan, se discuten. Sin embargo, esta actitud no dogmática está lejos de asemejarse a la actitud crítica del método científico.

Las normas contables obligan, facultan, recomiendan, una acción o comportamiento determinado. Tienen un componente normativo, denominado deóntico, y un componente fáctico, aquel hecho que es obligatorio, facultativo o recomendable. Si alguien ordena, "Debe calcular los costos por LIFO !", "debe" expresa el carácter deóntico, "calcular los costos por LIFO" el componente de hechos. Una norma contable elemental puede simbolizarse $O p$. El primer componente, $O$, es el carácter deóntico, por ejemplo, ser obligatorio. El segundo, $p$, describe un hecho, un proceso, una conducta. Analizaremos brevemente estos dos componentes.

El componente normativo expresa una función del lenguaje directiva. Esta función a diferencia de la informativa, cuyos enunciados pueden calificarse de verdaderos o falsos, no posee, o no se caracteriza, por los valores veritativos. Un enunciado directivo solamente incita o inhibe la acción. Si la orden "Debe calcular los costos por LIFO !" no se cumple, el enunciado no es falso. Solamente originará una sanción si quien la emite tiene suficiente poder. En otros términos, una norma no puede ser una hipótesis pues estas son enunciados informativos, por lo tanto, no se le puede aplicar el método científico.

Las normas al expresar una función directiva implican una lógica propia que incluye constantes lógicas específicas de esta función lingüística -obligatorio, facultativo, etc.- y también reglas de inferencia propias. Las lógicas deónticas se estudian desde hace largo tiempo, pero nunca se logró construir un sistema operativo (Wright 1979: especialmente caps. V y VIII). Así, perdemos la posibilidad de argumentar y sistemáticamente controlar la validez o invalidez de nuestros razonamientos al no tener aún una lógica deóntica operativa. De esta manera, o bien porque a las normas no se le puede aplicar el método científico, o bien por las limitaciones de la lógica deóntica, la contabilidad a priori queda en gran medida epistemológicamente disminuida.

El segundo componente son los enunciados fácticos. Los hechos pueden ser muy diversos, y en esta medida, una norma podría encapsular soluciones obtenidas según el primer modelo. Es decir, el 
componente fáctico podría consistir en una solución científica. Ahora bien, ¿puede una solución ser científica en la contabilidad a priori? No se la acepta con las condiciones de una hipótesis. No se extraen consecuencias elementales y entonces se las compara con la realidad. En la contabilidad a priori la discusión de las soluciones se asemeja a las discusiones ordinarias sobre deporte o política o asuntos familiares. Hay vagas conexiones con los hechos y se argumenta. Pero, para adoptar una solución tiene mayor fuerza la persuasión que la lógica y la realidad. La actitud crítica científica consiste en discutir enunciados comparándolos a través de sus consecuencias con la realidad, comparando soluciones diversas para determinar cuál describe mejor la realidad. Señalamos anteriormente que es la carencia de esta actitud crítica sistemática la que ha conducido a denominarla, con justicia, normativa o a priori.

Otra peculiaridad de estas soluciones contables es que sólo son aceptadas si son soluciones emitidas por organismos pertinentes de la profesión. Ellos son la autoridad emisora de las normas. No vale una solución distinta a la establecida hasta el momento que corporativamente sea reemplazada por otra. Este aspecto nunca lo encontraremos en las soluciones científicas. No puede decirse sino que resulta conservadora. En el campo científico las diferentes soluciones se libran a la competencia para determinar cuál es mejor. Metodológicamente ninguna puede demostrarse que es verdadera, y esto abre el campo al mejoramiento permanente de las soluciones. Dado una solución siempre hay un trabajo de articulación sin fin para mejorarla. Comparando el modelo científico con el contable, este último posee elementos que retardan el progreso.

¿Tiene fundamento científico la contabilidad normativa dejando de lado los aspectos legales? Taxativamente no. Es la principal razón por la cual se la ha denominado adecuadamente a priori o normativa. Sus enunciados se sostienen por razones ajenas al control empírico y a las relaciones lógicas entre ellos. Por su fundamento quedó relegada a la esfera de los más bajos tipos de acción, lo cual se refleja correlativamente en el descenso, respecto de la época de Pacioli, a los lugares inferiores en la jerarquía de saberes (ver Scarano 1997: 30). En síntesis, mientras las hipótesis se refieren a los hechos, las normas se refieren a la acción, están dirigidas a influenciar la conducta del contador. Las normas podrían llegar a encapsular soluciones científicas. En contabilidad, independientemente del éxito que tengan, es muy dificultoso mejorarlas por dos razones. La primera, que la aceptación o el cambio de normas, de soluciones, se produce corporativamente. La 
segunda, el sujeto de las normas es el contador, no puede ser la solución misma. Más importante aún, las soluciones no se imponen por su ajuste a los hechos. El modelo contable a priori no es anticientífico, pero es claramente precientífico.

Que el aspecto normativo no constituye ni condición necesaria ni suficiente de las soluciones sólidas -científicas- no es novedosa y en el continente americano dos destacados teóricos contables, entre otros, han sostenido esta posición: Richard Mattessich (1995, 2001), y Antônio Lopes de Sá (1998a, 1992).

DOBLE

\section{EL SISTEMA AXIOMÁTICO Y LA NATURALEZA DE LA PARTIDA}

\section{Estructura axiomática informal}

La formalización de la contabilidad permite discutir sobre las bases más estrictas posibles algunos aspectos metodológicos como la partida doble y la dualidad, el carácter empírico o normativo, la existencia de leyes. A continuación se presenta de una manera muy intuitiva una axiomatización contable (Ávila, González Bravo y Scarano 1990: cap. 4), el sistema $C$, que axiomatiza cualquier sistema contable por partida doble, es decir, contiene todos los términos y enunciados elementales necesarios para dar cuenta de los sistemas de registros contables. Luego, mediante extensiones de $C$, se amplía con axiomas adicionales para dar cuenta de variantes más específicas.

Una serie de definiciones facilita la exposición del sistema. Sea $V$ el conjunto de los valores contables registrados en un determinado período; en base a ese conjunto se produce la primera definición, cuenta, $C T$, una relación binaria cuyos argumentos son subconjuntos de $V$. La idea subyacente es que una cuenta tiene dos "partes" o "elementos", el debe y el haber de la cuenta que son subconjuntos de valores contabilizados. La segunda definición consiste en el conjunto de débitos de una cuenta. Naturalmente, consistirá en los elementos del conjunto que es el primer argumento de la relación cuenta. Esta relación se simboliza de la siguiente manera, CT $(x, y) ; x$, es el primer argumento e $y$, el segundo argumento. Los argumentos siempre son, por definición de la relación, subconjuntos de valores contables, entonces, el débito de una cuenta es el conjunto de valores contables que constituyen el primer argumento, $x$. La tercera 
definición, el conjunto de débitos de una cuenta se realiza de manera análoga, es el conjunto y, el segundo argumento.

Ahora resta someter a la relación $C T$ a restricciones específicas mediante axiomas para que funcione como un sistema contable:

Axioma 1 La unión de los conjuntos que constituyen los primeros elementos o argumentos de $C T$ es idéntico a $V$

Axioma 2 La unión de los conjuntos que constituyen los segundos elementos o argumentos de $C T$ es idéntico a $V$

Estos dos axiomas obligan a que la unión de los conjuntos del dominio y del codominio de la relación cuenta sea el mismo conjunto; en términos contables, el Debe es igual al Haber.

Axioma 3 Los conjuntos de débitos de las cuentas distintas son disyuntos (no tienen elementos en común)

Axioma 4 Los conjuntos de créditos de las cuentas distintas son disyuntos (no tienen elementos en común)

Estos dos últimos axiomas obligan a que un mismo valor no se acredite (debite) dos veces.

Axioma 5 Los conjuntos de débitos y créditos de una cuenta son disyuntos

Este axioma impide acreditar y debitar en una misma cuenta el valor de una operación registrada.

Una serie de definiciones suplementarias definen "d" y "h", el Debe y el Haber del Diario o del Mayor; "tk" el total de la sumatoria de los valores de un conjunto "k"; y "D" y "H", el conjunto de los saldos de cuentas deudoras y de cuentas con saldo acreedor, respectivamente.

De los axiomas enunciados y las definiciones expuestas, con ayuda de sencillas operaciones aritméticas, se siguen entre otros los siguientes teoremas: 


\section{Teorema 1 \\ $\mathrm{d}=\mathrm{h}$}

(El Debe y el Haber del Mayor contienen los mismos valores)

\section{Teorema 2 \\ $\mathrm{td}=\mathrm{th}$}

(El total del Debe y del Haber del Mayor son iguales)

\section{Teorema 3}

$\mathrm{tD}=\mathrm{tH}$

El sistema $C$ es una versión axiomática de cualquier sistema de registros contables por partida doble. Se pueden realizar extensiones suplementándolo con axiomas adicionales, por ejemplo, para generar un sistema de información lucrativa:

Axioma 6 Existe una partición dicotómica de $\mathrm{D}$, en $\mathrm{A}$ y Pe (Activo y Pérdidas)

Axioma 7 Existe una partición dicotómica de $\mathrm{H}$ en $\mathrm{Pa}$ y G (Pasivo y Ganancias)

Axioma 8 Existe una partición dicotómica de $\mathrm{Pa}$, en $\mathrm{Pt}$ y C (Pasivo para terceros y Capital)

$$
\begin{gathered}
\text { Teorema } 4 \\
\mathrm{tA}-\mathrm{tPt}=\mathrm{PN} \\
(\mathrm{PN}=\mathrm{tC}+\mathrm{tG}-\mathrm{tPe})
\end{gathered}
$$

Axioma 9 Existe una partición de $\mathrm{A}$ en $\mathrm{Di}, \mathrm{Cr}$, BV y $\mathrm{BU}$ (Disponibilidades, Créditos, Bienes para la venta y Bienes de uso)

$$
\begin{gathered}
\text { Teorema } 5 \\
\mathrm{tDi}+\mathrm{tCr}+\mathrm{tBV}+\mathrm{tBU}=\mathrm{tA}
\end{gathered}
$$

Axioma 10 Existe una partición dicotómica de Pe en CV y OG (Costo por Ventas y otros Egresos)

Teorema 6

$\mathrm{tCV}+\mathrm{tOG}=\mathrm{tPe}$ 


\begin{abstract}
Axioma 11 Existe una partición de Pt en Dt, Am, Pre y Pro (Deudas, Amortizaciones acumuladas, Previsiones y Provisiones)
Axioma 12 Existe una partición de $\mathrm{C}$ en $\mathrm{CP}, \mathrm{R}$ y $\mathrm{Ra}$ (Capital propio, Reservas y Resultados acumulados)

\title{
Axioma 13 Existe una partición dicotómica de G en IV y OI (Ingresos por ventas y Otros ingresos)
}

La función de los Axiomas 6-13 es asegurar la existencia de las clases que se necesitan para este tipo de contabilidad y así generar las conclusiones usuales como las exhibidas por los Teoremas 4, 5 y 6 . En general, al agregar axiomas adicionales a los Axiomas 1-5, se asegura la existencia de clases que permiten trabajar con un Plan de Cuentas específico.

¿Qué podemos inferir de esta presentación axiomática de la contabilidad? Por lo menos se destacan cuatro consecuencias. La primera, es la defensa tradicional de los desarrollos axiomáticos, una disciplina que lo consigue se expone de la mejor manera posible. Muestra sus puntos de partida tanto a nivel de conceptos como de enunciados. Es posible discutir y decidir si una conclusión se sigue o no en un sistema. Sólo de esta manera se puede elucidar cuáles supuestos que no pertenecen al campo propio son necesarios, la lógica supuesta y probar diversos resultados metateóricos.

La segunda, tiene que ver con el normativismo. Esta axiomatización muestra claramente que la esencia de la contabilidad -por ejemplo, el procesador contable- no consiste en normas sino en enunciados informativos. Visto de otra manera, si fueran normas estrictas ¿cómo aplicar a estos enunciados la matemática?

La tercera, permite discutir con mayor claridad el problema ¿cuál es la naturaleza de la contabilidad? ¿Se refiere a las conductas de los contadores o se refiere a la realidad contable? Otra manera distinta de preguntarse la misma cuestión es: ¿tiene leyes la contabilidad?, ¿a qué se refieren esas presuntas leyes o enunciados generales?

La cuarta, se conecta con la existencia de modelos contables alternativos. Muy fácilmente se advierte con esta presentación que existe un núcleo común a todos los modelos contables, los Axiomas 1- 
5 , y luego se obtienen diferentes modelos generados en base a diferentes objetivos, extendiendo el sistema en uno u otro sentido según los axiomas que se adicionan al núcleo común.

Una última observación respecto a la axiomatización expuesta: existen alternativas muy sofisticadas como la realizada por (Balzer y Mattessich 1991; 2000) en el marco de una visión estructuralista de las teorías; o la muy abarcativa de Lopes de Sá (1992, 1998a ó 1998b) que incorpora incluso aspectos de la gestión de una organización. Sin embargo, el sistema $C$ es suficiente para los objetivos propuestos.

2. La partida doble supone una estructura matemática que no se reduce a expresar la dualidad

A continuación examinaremos algunos teoremas de la aritmética bastante elementales, pero no por eso menos importantes para la contabilidad, pues expresan la partida doble, la dualidad típica que la caracteriza (Avila, González Bravo y Scarano 1990: 66-68).

$$
\mathrm{L}=\mathrm{M} \quad \begin{gathered}
T A \text { sólo } \mathrm{si} \\
\mathrm{tL}
\end{gathered} \mathrm{t}=\mathrm{tM}
$$

Este teorema afirma trivialmente que si dos conjunto de valores son iguales sus sumatorias también.

$$
L \cap M=\varnothing \quad \text { solo si } \quad \text { t } L+t M=t(L \cup M)
$$

El TA2 afirma que si dos conjuntos son disyuntos, no tienen elementos en común, entonces la suma de las sumatorias de dos conjuntos es idéntica a la sumatoria de la unión de ambos.

TA3

El pasaje de términos entre las sumas de las sumatorias de los elementos de dos particiones de clases idénticas, o de clases cuyas sumatorias son idénticas, preserva la identidad

Mostraremos una de las importantes aplicaciones de este último teorema clave.

Si $t D=t H$, y generamos las siguientes particiones de $D$ y $H$ respectivamente, $\{\mathrm{A}, \mathrm{Pe}\}, \mathrm{y}\{\mathrm{Pt}, \mathrm{C}, \mathrm{G}\}$, podemos obtener la unión de los elementos de cada partición, por hipótesis, 


$$
t(A \cup P e)=t(P t \cup C \cup G)
$$

aplicándole TA2 obtenemos:

$$
t A+t P e=t P t+t C+t G
$$

y por TA3 a esta línea,

$$
\mathrm{tA}-\mathrm{tPt}=\mathrm{tC}+\mathrm{tG}-\mathrm{tPe}
$$

En realidad, los Teoremas 2 y 3 arriba necesitan de algunos de los Teoremas TA precedentemente enunciados para demostrar explícitamente y de manera estricta que esos resultados se siguen de los axiomas (y la aritmética supuesta).

¿Qué aporta la explicitación de la aritmética supuesta? En primer lugar, la misma conclusión que en el apartado anterior respecto a la exposición de una disciplina -en este caso focalizando la atención en la matemática supuesta- desde un punto de vista formalizado.

La segunda, esta manera de presentar los aspectos cuantitativos, aritméticos, de la partida doble muestra los pasos a seguir para plantear con el mismo rigor y simplicidad la base cuantitativa para la partida triple, cuádruple, y así siguiendo.

La tercera, ilumina algunas concepciones que conciben a la contabilidad como matemática aplicada. El lado razonable de esta suposición es que efectivamente aplica estructuras matemáticas, y lo hace recurriendo a resultados no usuales, aunque elementales (la serie de Teoremas TA) que permiten expresar cuantitativamente la partida doble (o triple, etc.). Pero, ¿se reduce solamente a la aplicación de resultados matemáticos, se puede sostener que la contabilidad es solamente matemática aplicada? Claramente la respuesta es negativa: los Axiomas adicionales $A 6-A 13$ no son resultados matemáticos. Otra cuestión más básica aún, la relación $C T$ con las propiedades garantizadas axiomáticamente que enunciamos tampoco es una relación matemática. CT es una relación no lógica, un término descriptivo; estructura de tal manera los términos de su dominio y codominio que es posible a esos subconjuntos aplicarles los teoremas aritméticos señalados. Por estas razones, la contabilidad es más que matemática aplicada.

La cuarta, ¿esta aproximación brinda un indicio de las razones por las cuales Luca Pacioli ubicó la contabilidad como un apéndice de un tratado matemático? Hoy no se la expone de esa manera, y es razonable que no se lo haga por los motivos señalados en el párrafo inmediatamente 
precedente. En cambio, sería muy razonable lo que le indujo a hacerlo si captó la naturaleza matemática supuesta en la contabilidad (él era matemático), la naturaleza cuantitativa de la partida doble aunque no presentara la deducción explícita como una serie de Teoremas del tipo TA1-TA3.

La última, la más importante para nuestros objetivos, la dualidad solo es un aspecto de la partida doble ${ }^{1}$. Si la dualidad significa que todo registro se imputa dos veces, es decir, todo lo que se debita también se acredita y viceversa, implicaría trivialmente la identidad de ambos conjuntos, de los débitos y de los créditos, garantizados por los dos primeros axiomas. Sin embargo, la dualidad debe conformarse a un sistema de cuentas, como lo exigen los Axiomas 3 y 4 . De acuerdo a estos no podría haber una sola cuenta pues no se satisfarían. TA3 refuerza y generaliza lo anterior; es un resultado fundamental que no se podría obtener sin la combinación de la dualidad y un sistema de cuentas.

\section{EL ESTATUTO METODOLÓGICO DE LA CONTABILIDAD}

Las dos secciones anteriores han hecho referencia, si bien indirectamente, al estatuto de la contabilidad por su carácter normativo y su forma lógica. En esta sección el problema se trata explícitamente y, en particular, se examina si es una tecnología.

\section{La contabilidad como tecnología}

Se puede conceder que las soluciones a los problemas contables sean científicas, sin embargo, es muy dudoso que tengan el mismo estatuto que la física, la biología o la economía. La dificultad principal es mostrar leyes contables, es decir, la existencia de hipótesis generales muy bien contrastadas o "paradigmáticas" (según hablemos en términos metodológicos tradicionales o no). Sin embrago, para sostener la cientificidad de la contabilidad no es necesario exigir la existencia de leyes, basta mostrar que se comporta como una tecnología. De otra manera, se puede calificar de "científica" una disciplina o la solución a un problema si emplea el método científico o se busca la solución con su 
ayuda. Los resultados de la aplicación del método científico son la ciencia básica, la ciencia aplicada y la tecnología. La primera es la sistematización de leyes en los dominios de la física, la química, la biología, la economía, entre otras muchas. La segunda, básicamente, es la aplicación de las leyes de las ciencias básicas con un interés práctico. La tercera es una nueva clase de saber científico que se originó tardíamente, en el último tercio del siglo XIX. Consiste en la aplicación del método científico a problemas cuya solución se obtienen sin generar leyes propias, valiéndose del método científico y, de ser posible, de conocimientos suministrados por las ciencias básicas o formales. Las tecnologías más comunes son las ingenierías (ingeniería mecánica, electrónica, nuclear, etc.), las biológicas, de las cuales las dos con mayor impacto en el presente son la ingeniería genética y la medicina, y las sociales (planificación educativa, económica, la pedagogía, y -según se argumenta en este artículo- la contabilidad).

Siguiendo a Bunge (2004: cap. 11; 1985: cap. 5), las teorías tecnológicas comparten ciertos aspectos con las teorías científicas pues se basan en ellas. Los más importantes son: simplifican e idealizan la realidad a la que se refieren, es decir, la modelizan; incluyen conceptos teóricos; pueden absorber información empírica y realizar predicciones; son empíricamente contrastables.

Las teorías tecnológicas al estar vinculadas a la acción y formuladas a veces con la urgencia de conseguir resultados, se preocupan por los "efectos brutos" y controlables a escala humana. Las teorías se construyen lo más cercanas posible al nivel observable, se simplifican cuanto sea posible. Los plazos y los recursos limitados para realizar un proyecto vuelven las contrastaciones menos rigurosas, las predicciones más imprecisas, se asegura el resultado multiplicando coeficientes de seguridad. Las teorías tecnológicas son conceptualmente menos profundas. Sin embargo, pensadas desde los objetivos a conseguir son más ricas, se ocupan no solo de lo que es sino también de lo que debe ocurrir.

La tecnología está dirigida a la acción, sea a la realización de un artefacto como el diseño y planificación de una institución, sea a la modificación de un estado como en la adquisición de nuevas habilidades mediante capacitación. Las teorías básicas o aplicadas no están dirigidas a la acción, la tecnología las utiliza para diseñar, planificar y ejecutar acciones óptimas.

La acción puede encontrarse en un amplio espectro que va desde la acción refleja, la acción espontánea, la acción que supone una 
amplia acumulación de saberes y adquisición de experiencias y habilidades (la acción del experto o del artesano), hasta la acción tecnológica. Bunge intenta caracterizar la subclase de las acciones en la que está incluida la acción tecnológica definiendo la acción racional. Una acción es racional si cumple dos condiciones, la primera, la acción tiene que ser máximamente adecuada al objetivo propuesto; la segunda, tanto el objetivo como los medios para conseguirlo deben seleccionarse de acuerdo al mejor conocimiento disponible. Ahora bien, el conocimiento puede ser conocimiento común, experto, científico; lo cual obliga a considerar una subclase de los actos racionales, los máximamente racionales, es decir, aquellos que se basan en el conocimiento científico. La tecnología se interesa en estos actos.

La acción tecnológica produce o hace objetos estados 0 cambios con algún propósito. Otra manera de decirlo más sugestiva es que la acción tecnológica se basa en diseño y planes. Un diseño (tecnológico), o síntesis, es la representación de una cosa o proceso artificial anticipado con la ayuda del conocimiento científico. Una vez diseñado debe ser implementado mediante un plan. Un plan o planificación es una serie de ideas que describen operaciones o acciones, ejecutadas por seres racionales con el propósito de causar ciertos cambios especificados en las cosas. Un plan supone el problema inverso de la previsión. En esta última tenemos que conocer el estado final de un sistema; en el planeamiento está dado de antemano y debemos conocer qué estímulos y medios utilizar para llegar al estado final deseado a partir de un estadio inicial conocido.

El dominio de la tecnología no es la naturaleza sino el hombre, así formula y estudia reglas de acción no leyes de la naturaleza. Es fácil entender por qué los valores, y en particular un ethos está presente siempre en la tecnología, por qué los valores y en particular la ética no es externa a la tecnología sino interna. Esta es la interpretación subyacente en Mattessich (1995: chap. 11), cuando reivindica para la contabilidad una metodología condicional-normativa.

¿Cuáles son las consecuencias de considerar a la contabilidad una tecnología? La primera, se la puede calificar como "científica". Este no es un carácter meramente jerarquizarte frente a otros saberes o prácticas. Fundamentalmente hace referencia a la manera de resolver problemas y a cuáles soluciones son aceptables o no.

La segunda, se destaca inmediatamente la insuficiencia del normativismo. Primero hay que demostrar que una solución a la valuación, 
a la presentación de los estados contables, a la manera de imputar los costos, son soluciones contrastables y que han sobrevivido a este proceso. A lo sumo, el papel de las normas se reduce a encapsular soluciones sostenidas por otros criterios no normativos.

La tercera es de orden psicológico, pero no por esto menos importante. Muchos contadores sensatamente reaccionan contra la pretensión de considerar la contabilidad una ciencia básica como la física, la química o la biología. Si esto es cierto, la alternativa que queda no es reducirla a una mera práctica, a un arte. También se puede reivindicar un enfoque científico -tecnológico- para los problemas contables.

2. La contabilidad normativa es una disciplina única desde el punto de vista metodológico

Mostramos que el modelo contable a priori no es anticientífico, pero es claramente precientífico. Realicemos el siguiente experimento mental, imaginemos si la medicina, las ingenierías, la administración, la arquitectura, aún el deporte, y casi todos los cuerpos de praxis ligados a saberes, hubieran seguido el camino de la contabilidad a priori, es decir, como fundamento emitieran Principios de Ingeniería (Medicina-Administración-Arquitectura) Generalmente Aceptados y controlaran la actividad corporativamente... Como respuesta y como curiosidad señalemos que esta evolución de la contabilidad es única.

Las restantes disciplinas y prácticas ligadas al conocimiento desde principio de siglo paulatinamente se convirtieron o se asociaron a tecnologías. La contabilidad en soledad evolucionó sobre carriles opuestos. Al cabo de unas pocas décadas, sin éxitos que exhibir, con gran insatisfacción de los contadores, e incluso con desprestigio creciente para la contabilidad, ver como ilustración (Nelson 1973), comenzó a transitar en Estados Unidos el sendero prometedor por el cual tuvieron éxito las demás. La economía y la administración definitivamente se habían alejado tanto de la contabilidad que se invirtió la relación de la época de Pacioli. En ese entonces eran casi o totalmente inexistentes, la contabilidad era el saber respecto a los fenómenos económicos y reunía los rasgos más típicos de la nueva mentalidad moderna: antitradicionalismo, matematización, énfasis en al praxis creadora (cfr. Scarano 1997: 30).

La versión normativista extrema convierte a la contabilidad en un saber tan alejado de los cánones científicos que o bien, si se reduce 
a derecho se enseñaría en esa Facultad o directamente no tiene estatuto científico (no es ni ciencia ni tecnología) y debiera dejar de enseñarse en la universidad.

La alternativa al normativismo, desde el punto de vista metodológico, es la contabilidad científica, pero calificada de esta manera en virtud de su carácter tecnológico.

\section{CONCLUSIONES}

Se argumentó en favor del carácter lógico estándar, empírico y tecnológico de la contabilidad. Para demostrar el carácter fundamentalmente empírico primero se evaluó su aspecto fenomenológico más notable, la formulación mediante normas. El normativismo se manifiesta en distintas formas aunque posee varios rasgos comunes, el determinante, el carácter deóntico de sus enunciados; es decir, tienen una función directiva, no pueden desempeñar el papel de hipótesis.

Se señaló que el normativismo no fue un carácter permanente sino que tuvo su aparición con la crisis de las inversiones ferroviarias en Gran Bretaña y la actividad regulatoria surge para proteger al inversor; luego se extendió a otros dominios especialmente ante crisis económicas. Culmina a nivel teórico con el apriorismo contable en los setenta del siglo pasado, con creciente descontento y desconfianza acerca de la naturaleza de la disciplina así considerada.

Las normas no se sostienen porque se someten al control de la realidad sino por una justificación de otro orden. Si la contabilidad es exclusivamente normativa no puede ser científica, puede poseer ese carácter si el aspecto fundamental es el empírico y el secundario es el normativo.

La reconstrucción formal de la contabilidad implica enunciar los axiomas, básicamente exigen dualidad y caracterizan la noción de cuenta, y con axiomas adicionales se genera un Plan de Cuentas particular, por ejemplo, un sistema lucrativo. Se pueden demostrar una serie de lemas que garantizan las operaciones tradicionales, con estos resultados se obtienen como teoremas, entre otros, la tradicional ecuación patrimonial.

Esta formalización permite mostrar que el núcleo de la contabilidad no es normativo, por ejemplo, que para deducir la ecuación mencionada no se necesita un sistema normativo, ni ella misma lo es. 
También muestra las limitaciones de considerarla mera matemática aplicada. Las particiones necesarias para obtener clases y operarlas para finalmente obtener la ecuación patrimonial no dependen exclusivamente de características formales o matemáticas sino que también es esencial la dimensión empírica: ¿por qué realizar la partición del $D$ en $A$ y Pe? Formalmente podría ser cualquier otra, esa es necesaria en un sistema lucrativo porque cada una de ellas tiene significatividad empírica. Esas clases representan informaciones empíricas de la empresa relevantes para el fin deseado. Finalmente, la explicitación de la formalización permite fácilmente advertir cómo ampliar el sistema para generar otos modelos contables alternativos.

Considerar la contabilidad como una tecnología permite calificarla como científica y alude a la manera en que resuelve los problemas de su campo, la misma que las disciplinas científicas. Desde un punto de vista metodológico, el papel del regulacionismo en la teoría contable se reduce a lo sumo a encapsular soluciones científicas; en este sentido, antes que la norma debe estar la solución empírica al problema planteado -como es el caso de la tributación.

\section{BIBLIOGRAFÍA}

ÁVILA, H.E.; L. GonzÁlez BraVo y E.R. SCARANO (1990) "Una axiomatización de la contabilidad". L. González Bravo y E.R. SCARANo (1990) Aspectos metodológicos de la contabilidad. Buenos Aires: Eudeba-Impresos Centro, pp. 63-76.

BALZER, W. y R. MATTESSICH (1991) "An Axiomatic Basis of Accounting: A Structuralist Approach", Theory and Decision, 30, pp. 213-243.

- (2000) "Formalizing the Basis of Accounting". W. BALZER, J. SNEED and C.U. MOULINES (2000) Structuralist Knowledge Representation. Amsterdam: Rodopoy, pp. 99-126.

BUNGE, M. (2004) La investigación científica. Mexico DF: Siglo XXI Editores.

- (1985) Treatise on Basic Philosophy. Dordrecht and Boston: Reidel Publishing.

EDEY, Harold C. and P. PANITPAKDI (1956) "British Company Accounting and the Law 1844-1900". A.C. LITTLETON and B.S. YAMEY (1956) Studies in the History of Accounting. London: Sweet y Maxwell, pp. 356-379. 
LOPES DE SÁ, A. (1992) "Fundamentos lógicos de la teoría de las funciones del patrimonio hacendal", Cuadernos de Ciencias Económicas y Empresariales, 23, pp. 13-46.

- (1998a) Teoría general del conocimiento contable. Madrid: ICAC.

- (1998b) Teoria da contabilidade. São Paulo: Editora Atlas.

MATTESSICH, R. (1995) Critique of Accounting - Examination of the Foundations and Normative Structure of an Applied Discipline. London: Quorum.

- (1964; 2001) Contabilidad y Métodos Analíticos. Buenos Aires: La Ley.

MooNITZ, M. (1970) "Three Contributions to the Development of Accounting Principles Prior to 1930", Journal of Accounting Research, Spring, pp. 145-155.

NeLSON, C.L. (1973) "A Priori Research in Accounting". N. DOPUCH and L. REVSINE (eds.) Accounting Research 1960-1970: A Critical Evaluation, Monograph, No. 7, pp. 3-19.

PolLINS, H. (1956) "Aspects of Railway Accounting Before 1968". A.C. LITTLETON and B.S. YAMEY (1956) Studies in the History of Accounting. London: Sweet and Maxwell, pp. 332-355.

SCARANO, E.R. (1997) "La contabilidad y la aparición de la tecnología, ¿una involución?", IPAT- Boletim del Instituto de Pesquisas Augusto Tomelin, 12, fevereiro, pp. 25-38.

WRIGHT, G.H. von (1979) Norma y acción. Madrid: Tecnos.

ZEFF, S.A. (1972) "Chronology of Significant Developments in the Establishment of Accounting Principles in the United States, 19261972", Journal of Accounting Review, Spring, pp. 217-227. 